\title{
Role of biomarkers in nutritional science and industry - a comment
}

\author{
P. Weber* \\ F. Hoffmann-La Roche Ltd, Vitamins and Fine Chemicals Division, Research \& Development, \\ Human Nutrition and Health, CH-4700 Basel, Switzerland
}

\begin{abstract}
We are encountering a significant progress in nutritional knowledge, relevant to the practice of all aspects of human nutrition, medicine and public health. So, it is conceivable that we may view the role of nutrition differently in the future. The diet may not only provide an adequate amount of nutrients to meet the metabolic requirements, but could also contribute to improving human health status. As a consequence, extracts of plants or single compounds thereof which are believed to benefit human health need to be identified and developed for the food market to complement a balanced diet. The assessment of risk and benefit of constituents of a diet or plants will be a challenge for scientists working in this area. The number of compounds to be tested is enormous. Their impact on human health is supposed to be through prevention. Their effect on the human system may be modest, yet still significant when consumed over an entire lifespan. Trials employing traditional clinical endpoints, for many reasons, appear not to be feasible to investigate the relevance of these compounds on human health. Rather biomarkers, which are 'surrogate endpoints' for clinical events, may be used in the field of nutritional science. These biomarkers have to be highly sensitive and specific, non- or minimally invasive and inexpensive and they need to be validated and standardized. The biomarker concept may prove essential in nutritional science to demonstrate the effect of diet constituents on human health.
\end{abstract}

Biomakers: Nutritional science: Industry

\section{Background}

Demographics are changing dramatically in different countries all over the globe. In particular, in the Western World the population is ageing rapidly. In the United States (US) for example, the number of people aged 65 years and over is expected to double in the next decades. Americans older than 45 years will constitute almost one-half of the population (Hasler, 1996). These demographic changes will have important implications on both, the incidence of chronic diseases and health-care costs. On the other hand, we are encountering significant progress in nutritional knowledge, relevant to the practice of all aspects of human nutrition, medicine and public health. So, it is conceivable that we may view the role of nutrition differently in the future. Consumer expectations and demands of a diet may also change. In fact, there is evidence (Heasman, 1998) that the consumer may favour a diet, which not only provides an adequate amount of nutrients to meet the metabolic requirements, but can also contribute to improve human health status.

Actually, this process started some time ago. In the 1970s, scientists began to consider a diet high in fat and cholesterol as a risk factor for cardiovascular diseases. Subsequently, as appropriate foods became available in the marketplace people started to prefer low fat and cholesterol-free foods over conventional food, so as to minimize their cardiovascular risk. However, it appears that 'taking out bad things' (e.g. fat, sugar, etc.) from the diet is no longer good enough. To complement a balanced diet, scientists are now suggesting 'putting back or even enhancing the good things' (vitamins, polyunsaturated fatty acids, etc.) in the diet. As a consequence, extracts of plants or single compounds thereof, which are believed to benefit human health, need to be identified and developed for the food market.

\section{The challenge}

To make this happen appears to be quite a challenge for scientists in academia, in regulatory agencies and in industry. This comment is about the possible role and value of the biomarker concept in terms of nutritional science being carried out in industry.

Clearly, most of the extracts or single compounds of plants perceived to be healthy are basically nonessential and many do not have, what in the US is being referred to as a 'generally regarded as safe' (GRAS) status. So, first of all it has to be shown that these compounds are safe. Secondly, the putative health effect on the human body of a given compound has to

* Corresponding author: Dr P. Weber, fax +41 6168896 84, email peter.weber@roche.com 
be demonstrated by sound scientific experiments. Demonstration of efficacy is important to the industry, so a claim could then be made on a particular compound or product.

The assessment of risk and benefit of constituents of a diet or plants will be a challenge for scientists working in this area. The number of compounds to be tested is enormous. Furthermore, and even more importantly, the impact of a diet or single constituent on the human system may be modest at the time, yet may be significant when consumed over the entire lifespan. Plus, if these 'healthy' constituents are targetted against chronic diseases such as cardiovascular diseases or osteoporosis, there is another issue. Many chronic diseases may take a long time period to develop clinically overt symptoms (Hansson et al. 1994). For example, atherosclerotic alterations may be seen in situ in young adults or even earlier (Stary, 1987), yet clinical symptoms typically become evident only in later life. Also, for malignancies such as stomach cancer or prostate cancer it is known that from initiation of an aberrant cell to clinically overt cancer it may take 20 or more years. So, the inherent conflict of nutritional science is to demonstrate a significant effect of a given compound in a short amount of time, which very often will not resemble reality.

\section{Scientific tools}

The different categories of scientific evidence used to pinpoint an effect of a certain compound are in vitro tests (molecular/cellular assays), animal experiments, epidemiological studies and clinical trials. The definitive proof of a certain hypothesis, as generated in epidemiological studies, and further substantiated through in vitro assays and in animal experiments, eventually requires human clinical trials. The proof of efficacy in clinical trials, however, for the above reasons is problematic in nutritional sciences, when traditional clinical endpoints (i.e. myocardial infarction cancer incidence, fracture rate etc.) are being employed.

\section{Biomarker concept and nutritional science}

The biomarkers or 'surrogate endpoints' could be an opportunity to avoid that dilemma, when used instead of clinical endpoints in the field of nutritional science (Zeger,

Table 1. Definition of biomarkers, clinical endpoints and surrogate endpoints

\footnotetext{
- Biomarker

A characteristic that is measured and evaluated as an indicator of normal biological processes, pathogenic processes, or pharmacologic responses to an intervention

- Clinical endpoint

Variable that measures how a patient feels, functions or survives

- Surrogate endpoint

A biomarker intended to substitute for a clinical endpoint
}

From Zeger (1999).
Table 2. Examples of biomarkers and clinical endpoints

- Cardiovascular diseases

Biomarkers: serum cholesterol levels, blood pressure

Clinical endpoint: myocardial infarction, stroke

- Osteoporosis

Biomarkers: markers of bone remodelling

(formation/resorption), bone mineral density

Clinical endpoint: hip (spine) fractures

- Cancer, i.e. prostate

Biomarker: Prostate specific antigen

Clinical endpoint: neoplasm in the prostate or metastasis

1999), Table 1 lists the definition of biomarkers, clinical endpoints and surrogate endpoints as defined by Zeger (1999). These biomarkers have of course to meet some criteria to be useful. They have to be (1) highly sensitive and specific, (2) non- or minimally invasive, (3) validated and standardized, and (4) inexpensive. The biomarker concept may become an essential element in nutritional science to demonstrate the modest effect of nutrients or plant constituents on the human body within a reasonable period time and at reasonable costs. This applies, in particular to the development of compounds, which are intended to be used in food, so as to improve human health. Some examples of biomarkers relevant to nutritional science are given in Table 2. However, it must be realized that there are limitations to the biomarker concept. There will be no single biomarker which is ideal. The appropriate use of biomarkers may require a set of biomarkers aiming at the same indication. The result of these different biomarkers will generate a pattern, which will then be useful to assess the effect of a given compound on a certain condition or chronic disease. Not only will the result of the different markers always be unequivocal but also the different categories of scientific evidence such as epidemiology, molecular and cellular assays, animal experiments and eventually human trials will lead to results which have a different degree of scientific soundness. This may result in a grading of the scientific evidence that will be obtained from different studies. Scientifically solid results from clinical trials may be used to make a 'hard' claim, whereas associative data together with a set of preclinical data may only classify for a 'soft' claim (Fig. 1).

Soft claims

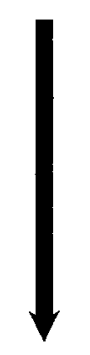

Molecular / cellular assays

Animal experiments

Epidemiology

Human studies

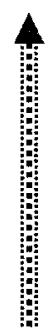

Hard claims

Fig. 1. Scientific evidence and claims. 
Also, the biomarker concept can be applied to test the safety of compounds. Traditionally, safety testing is carried out with animals and a set of clinical chemistry parameters is being assayed to assess the safety of that particular compound. The use of specific and sensitive molecular markers of organ damage, cellular and molecular signals as surrogates for tissue damage and eventually appropriate transgenic animal models may help to minimize animal testing. Finally, (molecular) biomarker approaches are useful while screening and identifying compounds which may potentially benefit human health.

\section{Conclusions}

The biomarker concept could facilitate a number of opportunities in academia, in regulatory authorities and in industry. For academia this concept could present a great research area to accelerate knowledge in nutritional science. A prerequisite to that is the validation of biomarkers. Clearly, collaboration between academia, regulatory authorities and industry is required, if this concept is to be successful. The interaction between academia, regulatory authorities and industry should be dynamic, in that knowledge will be applied as it becomes available and be replaced as there is superior information. From the industry perspective the biomarker concept could help to accelerate identification and testing of compounds, to develop a mechanism-based safety and efficacy concept of food constituents and to establish test models suitable for nutritional science. One of the key issues will be to translate biomarker-based scientific evidence into claims, which are required to successfully market a particular product.

In general, the biomarker concept may prove to be a fundamentally important element in research related to nutrition and public health. This may be a critical issue in the future as the demographics are expected to change dramatically within the next decade, so traditional public health services may face significant limitations.

\section{References}

Hasler CM (1996) Functional foods: The Western perspective. Nutrition Reviews 54, S6-S10.

Heasman MM (1998) The business of healthy eating. Financial Times Retails and Consumer. London: Financial Times Business Limited.

Hansson LE, Nyren O, Bergström R, Wolk A, Lindgren A, Baron J \& Adami HO (1994) Nutrients and gastric cancer risk. A population-based case-control study in Sweden. International Journal of Cancer 57, 638-644.

Stary HC (1987) Macrophages, macrophage foam cells, and eccentric intimal thickening in the coronary arteries of young children. Atherosclerosis 64, 91-96.

Zeger SL (1999) Biomarkers as surrogates in clinical research. NIH-Workshop. 